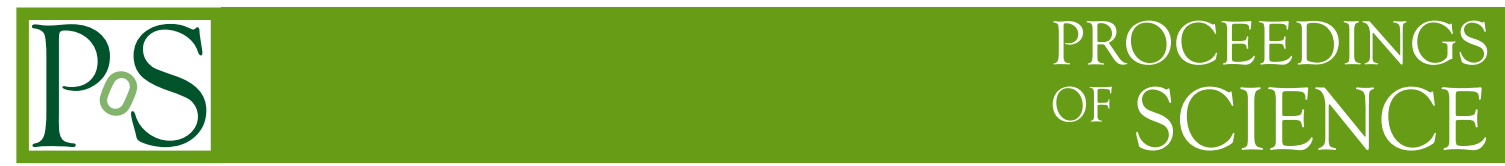

\title{
Nucleon Structure and Soft QCD from ATLAS
}

\section{André Sopczak ${ }^{a, *}$ on behalf of the ATLAS Collaboration}

${ }^{a}$ Institute of Experimental and Applied Physics, Czech Technical University in Prague, Husova 240/5, 11000 Prague, Czechia

E-mail: andre.sopczak@cern.ch

The ATLAS Collaboration has performed several analyses of nucleon structure and soft QCD. This review gives a concise summary of selected topics. New results with ATLAS W+jets and Z+jets data improve Quantum Chromo Dynamics proton Parton Distribution Functions. Single dissociative (soft $\mathrm{QCD}$ ) reactions and re-scattering are probed in photon-induced di-lepton production with a forward proton tag. A comparison of measurements with predictions of $g g \rightarrow \gamma \gamma$ production and dissociative reactions in light-by-light scattering and di-muon production in ultraperipheral $\mathrm{Pb}+\mathrm{Pb}$ collisions is presented. Inclusive single diffractive dissociation cross-section and a comparison with event generator predictions are reviewed. Properties of Underlying Event are determined by measuring charged-particle distributions in events containing $Z$ bosons decaying into a muon pair. There are various opportunities for co-operations with phenomenologists.

The Ninth Annual Conference on Large Hadron Collider Physics - LHCP2021

7-12 June 2021

Online

${ }^{*}$ Speaker 


\section{Impact of ATLAS W+jets and Z+jets data on proton Parton Distribution Functions}

Parton Distribution Functions (PDFs) are needed for accurate predictions of both Standard Model (SM) and Beyond Standard Model (BSM) cross-sections at the LHC. Using the ATLAS detector [1], a new set of proton PDFs, ATLASepWZVjet20, at Next-to-Next-to-Leading Order (NNLO) in QCD are provided [2]. The requirements for precise PDF determination are data covering a wide range of negative squared four-momentum transfer (denoted by $Q$ ) and Bjorken $x$, the fraction of the proton's longitudinal momentum carried by the parton initiating the interaction.

The results from ATLAS $\mathrm{W}+\mathrm{jets}$ and $\mathrm{Z}+\mathrm{jets}$ for $8 \mathrm{TeV}$ data $[3,4]$ were combined with ATLAS $7 \mathrm{TeV}$ data [5] and HERA data [6]. The PDF fit is performed at NNLO in perturbative QCD, which is made possible by recent theoretical developments for vector-boson production in association with jets [7, 8], and it accounts for correlations of systematic uncertainties between data sets. A comparison of the ATLASepWZVjet20 PDF set and an equivalent fit performed without the V+jets data PDF set (ATLASepWZ20) was given [2], which shows the improved agreement between data and the simulations using the new PDFs.

\section{Single dissociative events in photon-induced di-lepton production with a forward proton tag}

Scattered protons were detected by the ATLAS Forward Proton (AFP) spectrometer, while light-lepton pairs $(e e$ or $\mu \mu)$ were reconstructed in the ATLAS central detector for $\sqrt{s}=13 \mathrm{TeV}$ and $\mathcal{L}=14.6 \mathrm{fb}^{-1}$ [9]. The signal events result from exclusive or single dissociative (soft QCD) and rescattering reactions. A matching of lepton pair and proton kinematics, denoted by $\xi_{\ell \ell}$ and $\xi_{\mathrm{AFP}}$, is required (Figure 1, left). Data event candidates are illustrated in the di-lepton rapidity $y_{\ell \ell}$ versus $m_{\ell \ell}$ plane (Figure 1, right). The event selection includes a kinematic matching $\left|\xi_{\ell \ell}-\xi_{\text {AFP }}\right|<0.005$ on at least one side, where $\xi_{\ell \ell}$ is the expected proton energy loss based on lepton kinematics determined from di-lepton invariant mass and rapidity, and $\xi_{\mathrm{AFP}}$ is the fractional energy loss of the scattered proton [9]. Future double tag events will increase the distinction between exclusive/dissociated production.

In total $57(123)$ candidates in the $e e+p(\mu \mu+p)$ final states were observed. A backgroundonly hypothesis is rejected with a significance $>5 \sigma$ in each channel. Cross-section measurements in the fiducial detector acceptance $\xi_{\ell \ell} \in[0.035 ; 0.08]$ resulted in $\sigma(e e+p)=11.0 \pm 2.6$ (stat) \pm 1.2 (syst) \pm 0.3 (lumi) fb and $\sigma(\mu \mu+p)=7.2 \pm 1.6$ (stat) \pm 0.9 (syst) \pm 0.2 (lumi) fb.

From a comparison of these results with various models of soft survival probabilities, $S_{\text {surv }}$, it follows that re-scattering effects are important. Both the $m_{\ell \ell}$-dependent $[10,11]$ and fully kinematically-dependent [12] models describe the data satisfactorily.

\section{3. $g g \rightarrow \gamma \gamma$ production in light-by-light scattering and dissociative reactions in di-muon production in ultraperipheral $\mathrm{Pb}+\mathrm{Pb}$ collisions}

The observed signal is Light-by-Light (LbyL) scattering $\gamma \gamma \rightarrow \gamma \gamma$ [13]. The background reactions is Central Exclusive Production (CEP) $g g \rightarrow \gamma \gamma$. The photon Particle IDentification 



Figure 1: Left: Distributions of $\xi_{\mathrm{AFP}}-\xi_{\ell \ell}$ with $\xi_{\ell \ell}$ and $\xi_{\mathrm{AFP}}$ in the interval [0.02, 0.12] for side A and side $\mathrm{C}$. The total prediction comprises the signal and combinatorial background processes, where $p^{*}$ denotes a dissociated proton. The simulated predictions are normalized to data to illustrate the expected signal composition. The first (last) bin includes underflow (overflow). The hatched band indicates the combined statistical and systematic uncertainties of the prediction. Error bars denote statistical uncertainties of the data. Right: Data event candidates in the di-lepton rapidity $y_{\ell \ell}$ versus $m_{\ell \ell}$ plane satisfying event selection and kinematic matching, $\left|\xi_{11}-\xi_{\mathrm{AFP}}\right|<0.005$, on at least one side. Shaded (hatched) areas denote the acceptance (no acceptance) for the AFP stations indicated in the legend. Areas neither shaded nor hatched correspond to $\xi \notin[0,1]$. Taken from Ref. [9].

(PID) efficiency is optimized for low transverse energy $E_{\mathrm{T}}$. Distributions of di-photon acoplanarity and leading photon transverse energy show good agreement between data and signal plus background expectations.

The differential fiducial LbyL production cross-section in $\mathrm{Pb}+\mathrm{Pb}$ collisions was studied at $\sqrt{s}_{\mathrm{NN}}=5.02 \mathrm{TeV}$ and $\mathcal{L}=0.48 \mathrm{nb}^{-1}$. SUPERCHIC v3.0 [14] SM prediction gives a fair description of the data within uncertainties (experimental systematic and statistical, and theoretical). The statistical uncertainty on the CEP $g g \rightarrow \gamma \gamma$ production is $11 \%$ in the Control Region (CR) with negligible experimental uncertainties, and it changed to $21 \%$ with an extra gluon interaction and further uncertainties with varying parton distribution functions (PDFs) [13]. The CEP $g g \rightarrow \gamma \gamma$ background is $12 \pm 3$ events. In total, 97 events are observed in data where 45 signal events and 27 background events are expected.

\section{Inclusive single diffractive dissociation cross-section of pp collisions at $8 \mathrm{TeV}$}

The signal reaction is $P b P b(\gamma \gamma) \rightarrow \mu \mu(P b P b)$ [13], and the background reaction is dissociative $\mathrm{PbPb}\left(\gamma \gamma^{*}\right) \rightarrow \mu \mu+X\left(P b^{*} \mathrm{~Pb}\right)$. The data taken with the Zero Degree Calorimeter (ZDC) gives a total cross section of the $\gamma \gamma \rightarrow \mu \mu$ process in the fiducial volume of $\sigma_{\mu \mu}=$ $34.1 \pm 0.3$ (stat) \pm 0.7 (syst) $\mu \mathrm{b}$.

Events with a neutron tag on one side (Xn0n) and on both sides $(\mathrm{XnXn})$ as function of rapidity $\left|y_{\mu \mu}\right|$ are selected [13]. Good agreement of the shape with STARlight [15] prediction was observed, but STARlight generally tends to overestimate the fraction of events with forward neutrons [13].

\section{Inclusive single diffractive dissociation cross-section}

For this analysis, the ALFA Roman Pot stations in the outgoing LHC beams are used [16]. The data are recorded in special LHC runs with $\beta^{*}=90 \mathrm{~m}, \sqrt{s}=8 \mathrm{TeV}, \mathcal{L}=1.67 \mathrm{nb}^{-1}$ and with a low number of interactions per bunch crossing $\mu<0.08$. 
Single diffractive dissociation (SD) kinematics are described by the squared four-momentum transfer, $t$, mass, $m_{\mathrm{X}}$ of the dissociated system $\mathrm{X}$, and the proton energy loss $\xi$. The hadron-level cross-sections were studied versus $t, \xi$ and $\Delta \eta$. This was the first measurement of the $t$-spectrum in SD events at LHC [16]. The background events from non-SD $p p$ collisions have correlated signals in ALFA and the Inner Detector (estimated from MC), and overlay background with coincidences of a signal in ALFA with an uncorrelated signal in the Inner Detector (data-driven estimate, contributes the largest uncertainty).

The measured hadron-level differential SD cross-section shows the diffractive plateau as a function of $\Delta \eta$. An increase at small rapidity gaps is also noted (restricted rapidity region corresponding to the ATLAS tracker acceptance). A decrease at large rapidity gaps is visible due to fiducial range restrictions (loss of small- $\xi$ events close to the $\xi$-edge). The event generators PythiA8 and Herwig7 describe the shape reasonably, but overestimate the production cross-section.

Furthermore, the differential cross-section as function of $|t|$ was studied [16]. Pyтhia8 event generator predictions for $\mathrm{A} 2$ and $\mathrm{A} 3$ [17] are $B=7.82 \mathrm{GeV}^{-2}, B=7.10 \mathrm{GeV}^{-2}$, respectively. The corresponding measurement is $B=7.65 \pm 0.26$ (stat) \pm 0.22 (syst) $\mathrm{GeV}^{-2}$ [16].

\section{Underlying event in $\mathrm{Z}$ boson production}

Measurements of charged-particle distributions are sensitive to properties of Underlying Event (UE) in events containing $Z$ boson decaying into a muon pair $q q \rightarrow Z \rightarrow \mu \mu(\sqrt{s}=13 \mathrm{TeV}$ and $\mathcal{L}=3.2 \mathrm{fb}^{-1}$ ) [18]. The transverse thrust describes the event topology. Events with lower thrust are more sensitive to Multiple Parton Interactions (MPI). The background reactions are $Z \rightarrow \tau \tau$, $Z \rightarrow t t, W W \rightarrow \mu \nu \mu \nu$. Two transverse regions are differentiated by the scalar sum of the transverse momentum of the charged-particle: trans-max and trans-min. The trans-min region is highly sensitive to UE.

Powheg-Pythia8 has good agreement at low thrust, while HeRwig++ has good agreement at high thrust. SHERPa has overall good agreement [18].

In addition, the charge multiplicity versus the $Z$ transverse momentum was studied. Significant deviations were observed with respect to the generators predictions [18]. It is noted that the UE activity increases with $Z$ transverse momentum and center-of-mass energy.

\section{Conclusions}

ATLAS $\mathrm{W}+$ jets and $\mathrm{Z}+$ jets data improve QCD proton PDFs. Single dissociative (soft QCD) reactions and re-scattering were probed in photon-induced di-lepton production with a forward proton tag. Predictions and measurements were compared for $g g \rightarrow \gamma \gamma$ production, as well as dissociative reactions in light-by-light scattering and di-muon production in ultraperipheral $\mathrm{Pb}+\mathrm{Pb}$ collisions. Comparisons of inclusive single diffractive dissociation cross-section measurements with event generator predictions were performed. Differences in the recorded data description by Pythia8, Herwig++ and Sherpa are observed. The underlying events were measured in $Z$ boson production and compared to event generator predictions.

In the future, the precision will increase with the analysis of larger/new data sets. There is much potential for collaborations with phenomenologists. 


\section{Acknowledgments}

The project is supported by the Ministry of Education, Youth and Sports of the Czech Republic under the project number LTT 17018.

\section{References}

[1] ATLAS Collaboration, The ATLAS Experiment at the CERN Large Hadron Collider, JINST 3 (2008) S08003 [cds . cern. ch/record/1129811].

[2] ATLAS Collaboration, Determination of the parton distribution functions of the proton from ATLAS measurements of differential $W^{ \pm}$and $Z$ boson production in association with jets, JHEP 2107 (2021) 223 [2101.05095].

[3] ATLAS Collaboration, Measurement of differential cross-sections and $W^{+} / W^{-}$cross-section ratios for $W$ boson production in association with jets at $\sqrt{s}=8 \mathrm{TeV}$ with the ATLAS detector, JHEP 05 (2017) 077 [1711.03296].

[4] ATLAS Collaboration, Measurement of the inclusive cross-section for the production of jets in association with a $\mathrm{Z}$ boson in proton-proton collisions at $8 \mathrm{TeV}$ using the ATLAS detector, Eur. Phys. J. C 79 (2019) 847 [1907.06728].

[5] ATLAS Collaboration, Precision measurement and interpretation of inclusive $W^{+}, W^{-}$and $Z / \gamma^{*}$ production cross-sections with the ATLAS detector. Precision measurement and interpretation of inclusive $W^{+}, W^{-}$and $Z / \gamma^{*}$ production cross-sections with the ATLAS detector, Eur. Phys. J. C 77 (2016) 367 [1612.03016].

[6] ZEUS Collaboration, Combination of Measurements of Inclusive Deep Inelastic $e^{ \pm} p$ Scattering Cross-Sections and QCD Analysis of HERA Data. Combination of Measurements of Inclusive Deep Inelastic $e^{ \pm} p$ Scattering Cross-Sections and QCD Analysis of HERA Data, Eur. Phys. J. C 75 (2015) 580 [1506.06042].

[7] R. Boughezal, C. Focke, X. Liu and F. Petriello, W-Boson Production in Association with a Jet at Next-to-Next-to-Leading Order in Perturbative QCD, Phys. Rev. Lett. 115 (2015) 062002.

[8] A. Gehrmann-De Ridder, T. Gehrmann, E. W. N. Glover, A. Huss and T. A. Morgan, Precise QCD Predictions for the Production of a $Z$ Boson in Association with a Hadronic Jet, Phys. Rev. Lett. 117 (2016) 022001.

[9] ATLAS Collaboration, Observation and measurement of forward proton scattering in association with lepton pairs produced via the photon fusion mechanism at ATLAS, Phys. Rev. Lett. 125 (2020) 261801 [2009. 14537].

[10] L. A. Harland-Lang, V. A. Khoze and M. G. Ryskin, The photon PDF in events with rapidity gaps, Eur. Phys. J. C 76 (2016) 255. 
[11] M. Dyndal and L. Schoeffel, The role of finite-size effects on the spectrum of equivalent photons in proton-proton collisions at the LHC, Phys. Lett. B 741 (2015) 66.

[12] L. A. Harland-Lang, M. Tasevsky, V. A. Khoze and M. G. Ryskin, A new approach to modelling elastic and inelastic photon-initiated production at the LHC: SuperChic 4, Eur. Phys. J. C 80 (2020) 925.

[13] ATLAS Collaboration, Exclusive dimuon production in ultraperipheral $\mathrm{Pb}+\mathrm{Pb}$ collisions at $\sqrt{s_{N N}}=5.02 \mathrm{TeV}$ with ATLAS, Phys. Rev. C 104 (2021) 024906.

[14] L. A. Harland-Lang, V. A. Khoze and M. G. Ryskin, Exclusive LHC physics with heavy ions: SuperChic 3, Eur. Phys. J. C 79 (2019).

[15] S. R. Klein, J. Nystrand, J. Seger, Y. Gorbunov and J. Butterworth, STARlight: A Monte Carlo simulation program for ultra-peripheral collisions of relativistic ions, Comp. Phys. Comm. 212 (2017) 258.

[16] ATLAS Collaboration, Measurement of differential cross-sections for single diffractive dissociation in $\sqrt{s}=8 \mathrm{TeV}$ p p collisions using the ATLAS ALFA spectrometer, JHEP 2002 (2019) 042 [1911.00453].

[17] ATLAS Collaboration, The Pythia 8 A3 tune description of ATLAS minimum bias and inelastic measurements incorporating the Donnachie-Landshoff diffractive model, ATL-PHYS-PUB-2016-017 (2016) .

[18] ATLAS Collaboration, Measurement of distributions sensitive to the underlying event in inclusive Z-boson production in pp collisions at $\sqrt{s}=13 \mathrm{TeV}$ with the ATLAS detector, Eur. Phys. J. C 79 (2019) 666 [1905 . 09752]. 